\title{
Analysis of Completeness and Timeliness of Death Notification Data in Thika Level Five and Maragua Level Four Hospitals, Kenya
}

\author{
Dennis Mwenda ${ }^{1 *}$, Zakayo Thaimuta $^{2}$ \\ 1. Forensic Medicine Department, School of Clinical Medicine, Mount Kenya University, P. O. Box \\ 59360-00100 Nairobi, Kenya \\ 2. Department of Human Pathology, School of Medicine, University of Nairobi, \\ P. O. Box 19676 - 00202, Nairobi, Kenya \\ * E-mail of the corresponding author: mwendadeno@gmail.com
}

\begin{abstract}
Death notification is important in the service of justice to the victims of crime, punishment of criminals, preparation of forensic policies, guidelines, regulations and planning. This data is captured in mandatory forms in a prescribed format. Death notification form (DNF) is one of the prescribed forms to record and report death. One of the commonly observed setbacks concerning this form is the turnaround time and omissions.

The objective of this study was to analyse the completeness and timeliness of death notification data in Thika Level Five and Maragua Level Four Hospitals. The study was a retrospective review of records and a crosssectional survey conducted at Thika Level Five and Maragua Level Four Hospitals and civil registration departments in Murang'a and Kiambu Counties. We recruited fifty one clinicians (25 from Thika and 26 from Maragua hospitals) who are involved in filling the DNF. All the DNF certified by a qualified health professional at Thika Level Five and Maragua Level Four Hospitals for the year 2017 and filed with Civil Registrar of the respective counties were sampled for this study. Two thousand two hundred and forty four death notifications form (Thika Level Five, 1524 and Maragua Level Four, 720 Hospitals) and were accessed and an analysis on their completeness was done.

It was found out that most of the death notification forms: $75 \%$ in Thika Level Five and 62\% for Maragua Level Four hospitals were not filled within 48 hours and 14\% were left blank in both centres. This study revealed that $78 \%$ of death notification forms (DNF) were filed comprehensively. The common missing information on the DNF was information on underlying cause of death (35\%) and source of the data used to fill the death notification forms $(32 \%)$. There was a statistically significant relationship between the hospital where the forms were filled and timeliness in filling the forms $\left(\chi^{2}=35.433, \mathrm{p}=0.001\right)$. Regression analysis results revealed that hospital where the records are reported $(\mathrm{p}=0,001)$, qualifications of the certifier of forms $(\mathrm{p}=0.022)$, age of the deceased $(\mathrm{p}=0.014)$, gender of the deceased $(p=0.015)$, underlying cause of death $(p=0.001)$, intermediate cause of death $(p=0.001)$, immediate cause of death $(0.001)$ and source of the information used to fill the death notification forms $(p=0.001)$ were statistically significant compared with completeness of the DNF.

Most facilities lack guidelines, trainings and provision of feedback on the filling of the death notification forms which affects their completeness and timeliness in filling. In all the studied hospitals it was concluded that it is necessary to harmonize how the death notification forms are filled in the hospitals. Clinicians who attend formal pre-training sessions have higher knowledge in filling the forms than those who have not been sensitized. There is no formal training or sensitization in both hospitals on DNF.
\end{abstract}

Keywords: Autopsy; Completeness; Death notification

DOI: $10.7176 / \mathrm{JLPG} / 101-22$

Publication date:September 30th 2020

\section{Introduction}

According to United Nation, (2004) monitoring and socio-economic planning requires data on both the number of deaths and the causal arrangements that resulted to mortality. The ideal approach to help the living is by checking the mortality and their causality. Cause of death information gives experiences in the diseases, way of life issues and outside elements adding to decreased future. Many countries don't utilize the international classification of disease (ICD) tenth amendment created by the World Health Organization (United Nations, 2004). This body has established the international standard analytic classification used to group cause of death among other wellbeing parameters (United Nations, 2004). There has been a huge advance in the quantity of nations that have National Strategies for the Development of Statistics (NSDS). Extensive learning on the requirement for secure and classified sharing of individual data crosswise over government organizations civil registration and fundamental measurements. Thereby death being a constant and permanent event, its certification should be actual and true to hold its relevance in statistics. Studies have established considerable misclassification on the cause of death of 
individuals who passes on in doctor's facilities with chaperon suggestions for the utilization of cause-of-death information in advising arrangement (Yudkin et al, 2009). A symposium held at Kenyatta National Hospital (KNH) in conjunction with University of Nairobi in 2016, Theme "Therapeutic legitimate issues in wellbeing", it was submitted that there is a genuine test in the filling and finishing death notification information in Kenya by health workers. This study was purposed to examine for completeness and timeliness of death notifications collected in local hospitals to identify challenges and highlight possible solutions.

\section{Materials And Methods}

The study was both a retrospective by reviewing death notification forms which were filled on January 2017 through December 2017 and a cross-sectional descriptive study by involving the clinicians responsible for filling the death notification forms at the two hospitals. Descriptive study was used to describe the variables collected such as level of completeness and timeliness of death notification forms and characteristics of reviewed forms and study participants.

The study population comprised 51 clinicians (25 from Thika and 26 from Maragua hospital) and 2,244 death notification forms in Thika Level Five hospital and Maragua Level Four Hospitals between Januarys to December 2017 whose death notification forms were available in the office of the civil registrar. Permission to conduct this study was sought from Mount Kenya University Ethics Committee (MKU/ERC/0541) and consent to do this study was obtained from Thika Level Five and Maragua Level Four Hospitals. Research permit was obtained from National Commission for Science, Technology and Innovations (NACOSTI/P/17/60783/19736). Consent to abstract data from death notification forms was obtained from the Office of the Civil Registrar (CRD/ADM/149/Vol. III (35).

The death notification forms were sampled based on deaths occurring within the hospital setup and were certified by a qualified health professional. Taro Yamane formula (ZaherNazzal 2017) was used to calculate the sample size for the study.

In the office of civil registrar, DNF from the hospitals are filed based on where the death occurred, this being the ward where the deceased was last admitted before death happened. The wards where deaths were reported were categorized as: obstetrics and gynaecology, medical, paediatrics, casualty and surgery. Therefore, stratified random sampling was used to sample the death record forms for review from each of the two hospitals based on five categories of place. The five categories used to classify place of death were used as strata for sampling. In each of the strata, an Excel list of all notification records which meet the inclusion criteria were compiled using a continuous numbering (coding) system in each of the hospitals. Using this list, a proportionate sample of forms from each of the stratum was randomly sampled for review founded on the total number of deaths recorded in each of the strata. Using this sampling design, a total of 339 records; comprising of 230 forms from Thika level five Hospital and 109 forms from Maragua level four Hospital were stratified randomly sampled and reviewed in Thika and Maragua hospitals respectively. Data was analysed using IBM SPSS statistics. The Statistical significance was at $\mathrm{p}<0.05$.

\section{Results}

Table 1: Proportionate Distribution of Sample Size for Form Reviews

\begin{tabular}{llll}
\hline Hospital & Stratum & Total No. of Forms & Sample \\
\hline Thika Hospital & Obstetrics and gynaecology & 69 & 10 \\
& Paediatric & 244 & 40 \\
& Medical & 770 & 76 \\
& Surgery & 221 & 52 \\
& Casualty & 221 & 52 \\
Total & Obstetrics and gynaecology & $\mathbf{2 3 0}$ & 0 \\
& Paediatric & 0 & 23 \\
& Medical & 1524 & 26 \\
& Surgery & 281 & 35 \\
& Casualty & 230 & 25 \\
\end{tabular}


From the findings, $67 \%(n=228)$ of the reviewed forms were from Thika Level Five Hospital while $33 \%(n=111)$ were reviewed from Maragua Level Four Hospital, table 1.

\section{Timeliness Documentation}

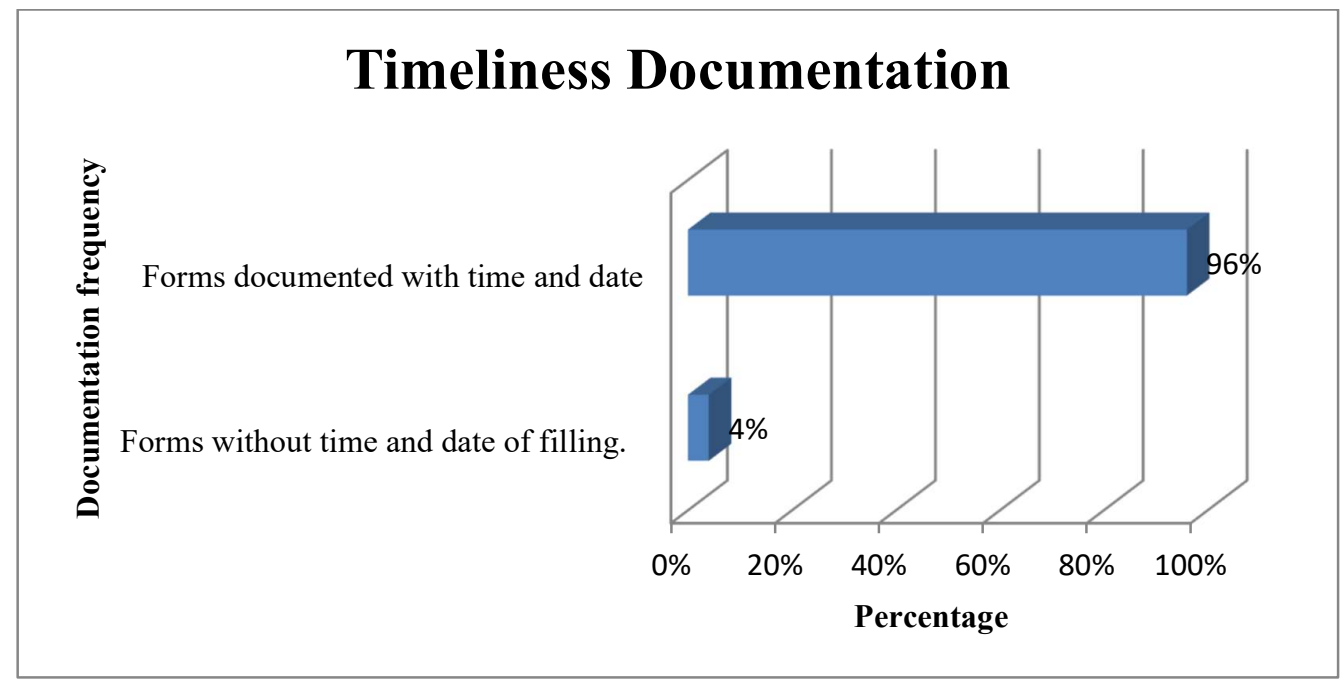

Figure 1: Timeliness in filling death notification

From the review, $96 \%(n=325)$ forms were documented time and date of filling. Out of this, only $29 \%(n=94)$ were timely filled within 48 hours. The DNF not completed within 48 hours period after death at Thika Level Five was at $75 \%(n=244)$. The DNF with undocumented time and date from Maragua Level Four Hospital was $4 \%(n=14)$. Out of the 339 forms retrieved for review at Maragua Level Four Hospital, 96\% $(n=325)$ were timely documented, figure 1 .

\section{Completeness of death notification forms}

Completeness of a death notification forms was measured by the proportion of mandatory fields filled in the forms by the clinicians. According to the forms, the clinicians are required in the minimum to fill ten fields of the forms namely: (i) name of diseased, (ii) age, (iii) date/time of death, (iv) place of death, (v) immediate cause of death, (vi) intermediate cause of death, (vii) underlying cause of death, (viii) source of information used to fill the forms, (ix) name of the clinician and (x) the title of the clinician. Findings showed that the DNF completely filled accounted for $78 \%$ overall. Completeness level was comparable for the two hospitals as shown in figure 2 .

\section{Completeness level of the forms}

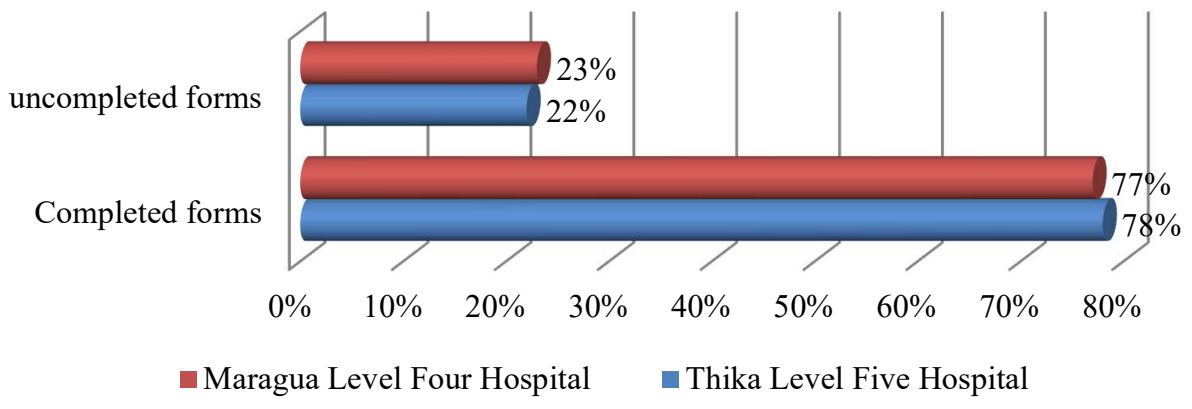

Figure 2: Completeness of death notification 
Similarly, uncompleted DNF for Thika Level Five Hospital accounted to 22\% ( $n=50)$ while Maragua Level Four Hospital uncompleted forms accounted to $23 \%(n=26)$, figure 2.

To establish factors associated with timeliness of filling the DNF, cross-tabulation and tests of independence (Fisher's exact test and chi-square tests) were done and results tabulated in Table 2. In regard to hospital where death is recorded, there was a substantial difference in timeliness of filling death notification forms in the two hospitals studied. Thika Level Five hospital had the highest rate of untimely filling of death notifications $(75 \%)$ compared to $25 \%$ documented in Maragua Level Four hospital. There was a statistically significant relationship between the hospital where the forms were filled and timeliness in filling the forms $\left(\chi^{2}=35.433, p=0.001\right)$. In regard to certifiers, highest proportion of forms filled within the required 48 hours were filled by Medical Officer intern MOI (69\%) followed by pathologists $(15 \%)$. Medical Officers have the least rate of timeliness in filling the death notifications. The qualification for the DNF certifier has a statistically significant relationship with timeliness in filling the forms $\left(\left(\chi^{2}=15.623, \mathrm{p}=0.035\right)\right.$.

Linear regression was used to analyse the relationship between factors and level of completeness (the continuous outcome variable) of the death notification forms as summarized in Table 2 . The regression analysis results shows that hospital where the records are reported $(\mathrm{p}=0,001)$, qualifications of the certifier of forms $(\mathrm{p}=0.022)$, age of the deceased $(\mathrm{p}=0.014)$, gender of the deceased (0.015), underlying cause of death $(0.001)$, intermediate cause of death (0.001), immediate cause of death (0.001) and source of the information used to fill the death notification forms (0.001) has a statistically significant relationship with completeness of the DNF.

Table 2: Factors associated with completeness of the notification forms

\begin{tabular}{lllll}
\hline \multirow{2}{*}{ Variable } & \multicolumn{2}{l}{ Unstandardized Coefficients } & \multirow{2}{*}{ P-value } \\
\cline { 2 - 4 } & Beta & Std. Error & \\
\hline Hospital & 1.773 & .531 & -3.343 & .001 \\
Department & .079 & .165 & .479 & .633 \\
Certifier & .632 & .274 & 2.302 & .022 \\
Age & .807 & .327 & -2.466 & .014 \\
Education Level & .038 & .160 & .234 & .815 \\
Sex & 1.175 & .479 & 2.456 & .015 \\
Single cause per line & .174 & .635 & -.274 & .784 \\
Correct Sequencing of death & .207 & .840 & -.246 & .806 \\
Underlying cause & 3.916 & .193 & 20.251 & .001 \\
Intermediate cause & 3.785 & .199 & 19.021 & .001 \\
Immediate cause & 8.903 & .509 & 17.494 & .001 \\
Information source & 11.313 & .464 & 24.397 & .001 \\
(Constant) & 19.884 & 2.374 & 8.376 & .001 \\
\hline & & & & \\
\hline
\end{tabular}




\section{Knowledge level of clinicians}

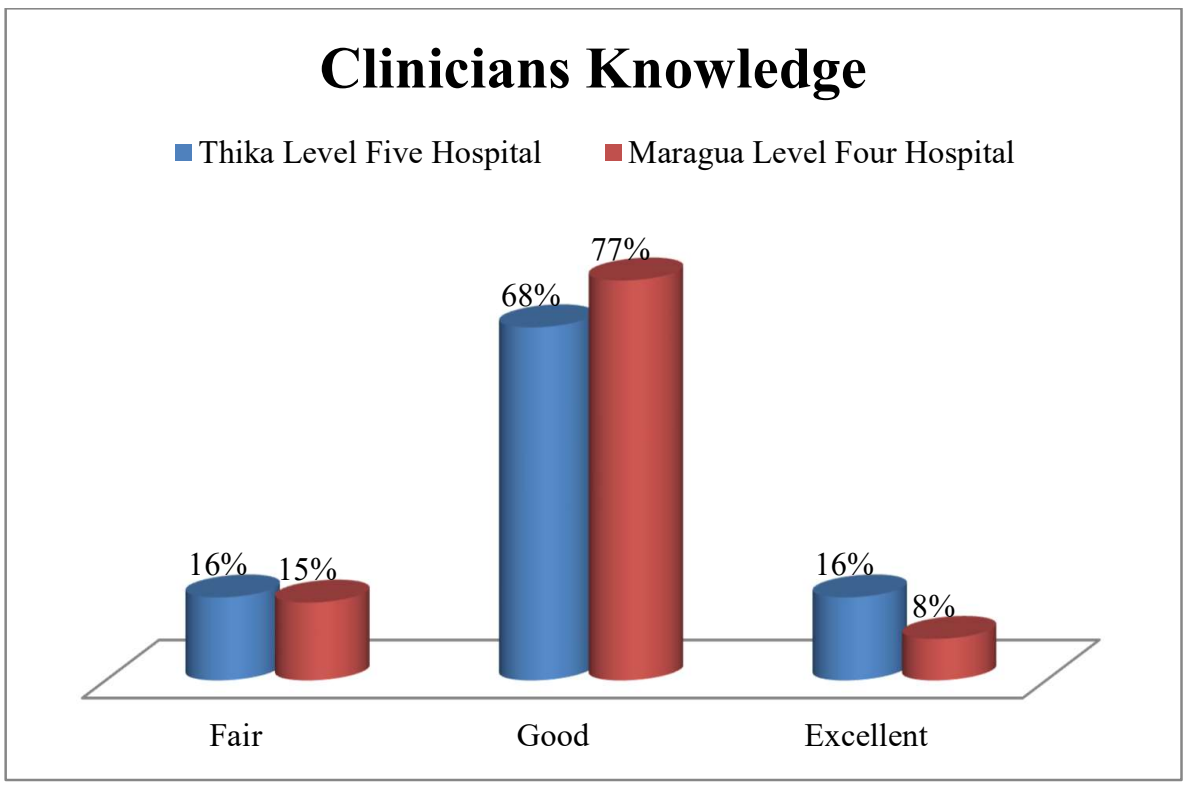

Figure 3: Clinicians Knowledge level

The clinicians in both Maragua Level Four Hospital and Thika Level Five Hospitals rated their knowlede for DNF as good, accounting to $77 \%(\mathrm{n}=20)$ and $68 \%(\mathrm{n}=17)$ respectively while those who rated their knowledge as excellent, accounted to $16 \%(\mathrm{n}=4)$ from Thika Level Five and $8 \%(\mathrm{n}=3)$ from Maragua Level Four Hospitals. Those who rated their knowledge as fair, accounted to $16 \%(n=4)$ and $15 \%(n=4)$ for Thika Level Five and Maragua Level Four Hospitals respectively, figure 3.

Clinicians' qualification and knowledge relation

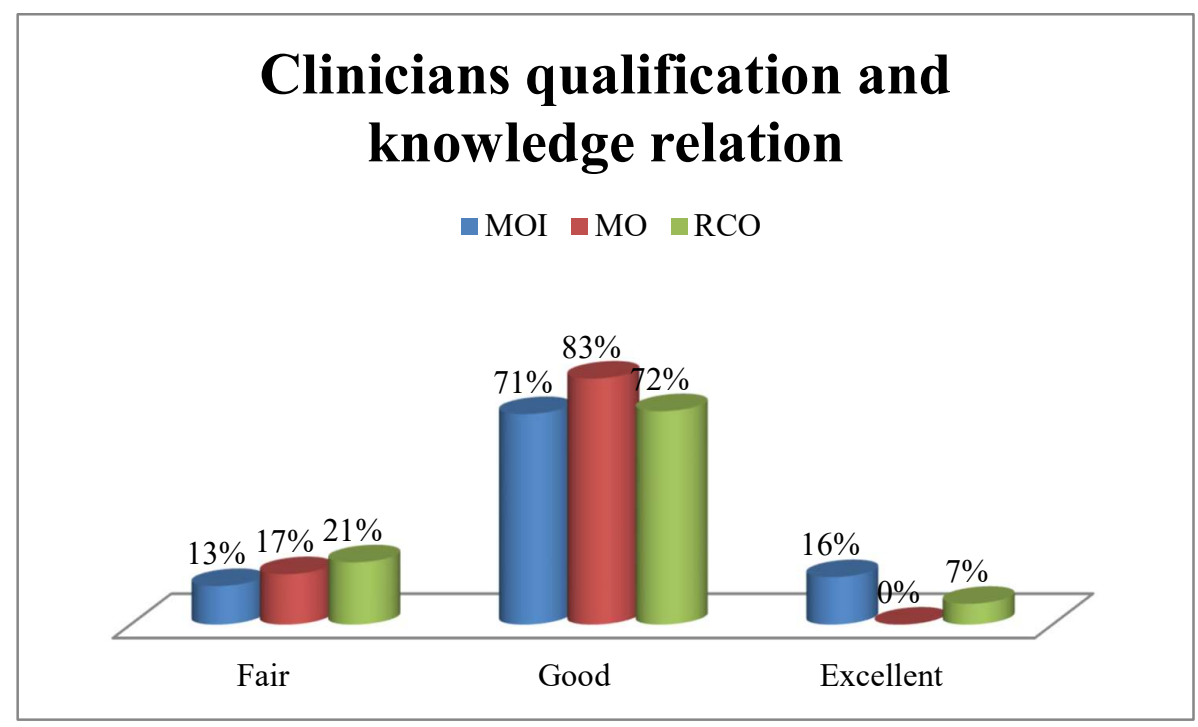

Figure 4: Relationship between qualification of clinicians and their knowledge level

From the findings, majority of clinicians interviewed rated their qualification to knowledge as good, accounting to $71 \%(n=22)$ for MOI, $83 \%(n=5)$ for $\mathrm{MO}$ and $72 \%(n=10)$ for $\mathrm{RCO}$ while those who rated their relation as 
excellent, accounted to $16 \%(\mathrm{n}=5)$ for MOI and $7 \%(\mathrm{n}=1)$ for RCO. Those rated their relation as fair, accounted to $13 \%(n=4)$ for MOI, $17 \%(n=1)$ for MO and $21 \%(n=3)$ for RCO, figure 4 .

Pre-trained and Non pre-trained knowledge level

\section{Pre- trained and Non pre-trained knowledge Level}

Non Pre-trained $\quad$ Pre-trained

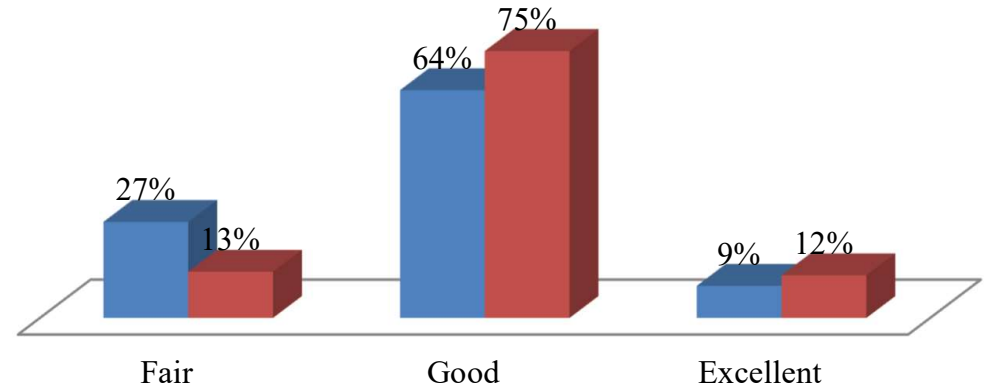

Figure 5: Relationship between pre-training and their knowledge level

Knowledge level was rated as good overall $75 \%(\mathrm{n}=30)$ among the pre-trained and Non pre-trained was $64 \%(\mathrm{n}=7)$. Knowledge was rated fair by $27 \%(\mathrm{n}=3)$ and $13 \%(\mathrm{n}=5)$ both Non pre-trained and pre-trained respectively. Knowledge level was rated excellent by $9 \%(n=1)$ and $12 \%(n=5)$ by Non pre-trained and pre-trained respectively, figure 5 .

Availability of Guidelines, Seminars and Feedbacks on Death Notifications

\section{Availability of seminars, feedacks and guidelines}

m Thika Level Five Hospital a Maragua Level Four Hospital

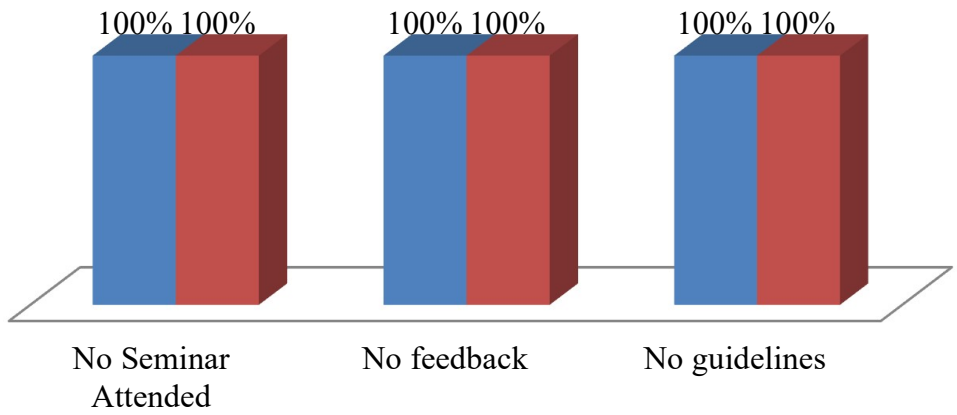

Figure 6: Attendance of seminars and provision of feedback on the notification forms 
It was established that clinicians from both Thika Level Five and Maragua Level Four Hospitals attended no seminars, feedbacks or had any guidelines concerning quality of DNF, figure 6 .

\section{Clinician Perceptions on Filling of Death Notification Forms}

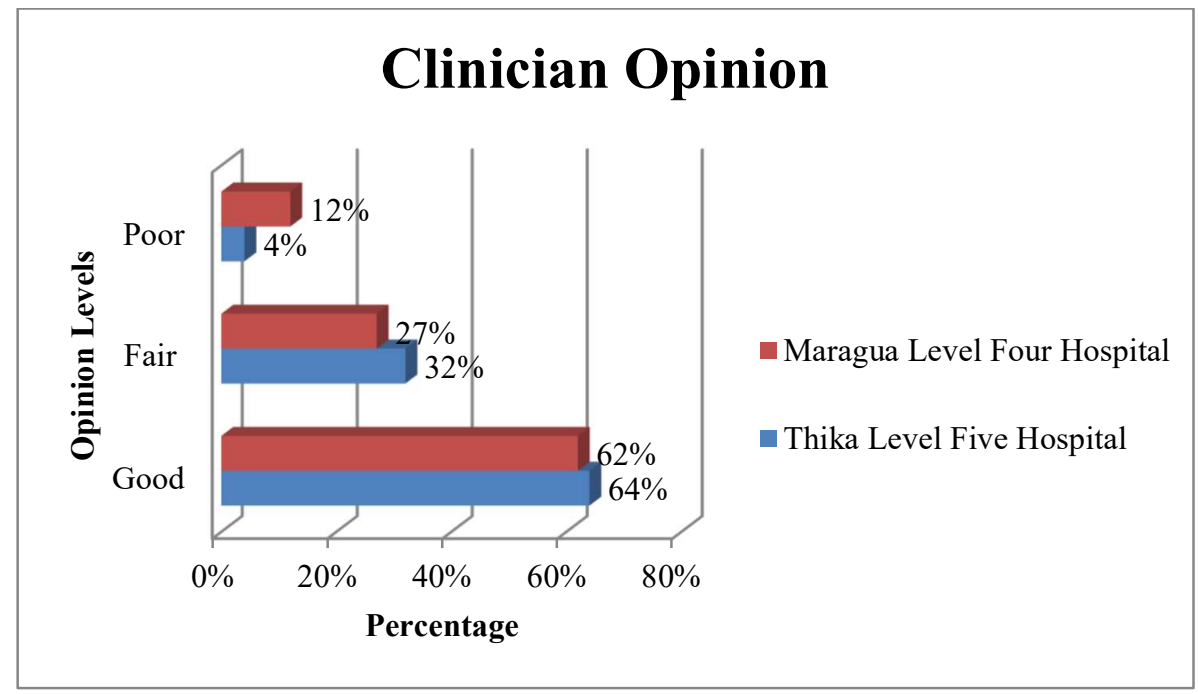

Figure 7: Clinician opinions on death notification forms filling

From the findings, over half of respondents rated opinions on DNF filling as good, were comparable in the both study sites: $64 \%(n=16)$ and $62 \%(n=16)$ for Thika Level Five and Maragua Level Four Hospitals respectively. Those who rated opinion as fair, accounted for $32 \%(n=8)$ and $27 \%(n=7)$ for Thika Level Five and Maragua Level Four Hospitals respectively. Those who rated opinion as poor accounted to $4 \%(n=1)$ for Thika Level Five and $12 \%(n=3)$ for Maragua Level Four Hospitals, figure 7.

\section{Discussion}

The study found out that a majority of forms were not filled on time $75 \%$ in Thika level four hospital and $62 \%$ for Maragua sub county hospital and surprisingly $14 \%$ overall were left blank. Indication of time of death is fundamental in provision of wholesome picture in determination of the sequence of events leading to death. These results were consistent with two studies carried out in South Africa in 2007 and 2009 which had absence of time interval reporting 81.5 and $98.4 \%$ respectively (Yudkin et al, 2009). Showing the time estimate for each condition in the sequence of events is fundamental in provision of a wholesome picture in determination of the underlying cause of death. Health workers must be keen to these entries as they portray the chronology of happenings that gives a vital indicator on the sequence of morbidities that can avoid errors of improper sequencing (ZaherNazzal, 2017).

According to the development agenda after 2015, timely statistics on deaths as a result of act of registration was key in monitoring progress towards sustainable development goals (WHO, 2017). This study also found out that $32 \%$ of the analysed death notification forms were blank on the source of information for filling the death notification form. It could not be established whether the clinician attended to the patient before death, examined the dead body or performed a post mortem to ascertain the cause of death.

This is fundamental especially in deaths of forensic interest whereby the clinician must demonstrate at what point the person died and what parameters were applied to confirm this event of which almost all are dependent on time. This study revealed a 78\% level of completeness. A substantial fraction of analysed DNF was found to have incomplete data. Overall, the underlying cause of death was missing in $35 \%$ and source of information filled missed on $32 \%$ of DNF. 
This compares to a study made in North West bank Palestine by Jamal (2017) whose findings were 99\% incorrect clinical sequence. Clearly this depicts inaccuracy in filling the DNF. It also compares well to a study made in $35 \%$ (WHO, 2000). Reporting the mechanism of death without its underlying cause is of grave concern that compromises the quality of DNF and therefore minimizes its benefit. This study further established that the intermediate cause of death was missing in some of the DNF analysed. This is necessary in that it gives the picture of how a certain underlying cause lead to loss of life for the end users of DNF (mainly the lawyers and judges) have limited clinical knowledge.

Completely filled DNF is utilized in the identification of the deceased and easy tracking of major events which the deceased was involved in that could create can link a suspect to other crimes or incidents. Substantial difference in timeliness of filling death notification forms in the two hospitals was demonstrated in this study. This is associated with the qualification of clinicians filling the DNF in that MOI only fill this document at Thika and Maragua has other cadres filling. Thika Level Five had the highest rate of untimely filling of DNF compared to Maragua Level Four hospitals $(p=0.001)$. This was attributed to number of deaths in the facility and the requirement that DNF must be filled. In regard to certifiers, highest proportion of forms filled within the required 48 hours were filled by MOI (69\%) followed by pathologists (15\%). MOs have the least rate of timeliness in filling the death notifications. The qualification of the death notification form certifier has a statistically significant relationship with timeliness in filling the forms $((\chi 2=15.623, \mathrm{p}=0.035)$.Evidence studies have shown that Kenyan doctors hardly get adequate opportunities to learn about death notification as part of medical training (Institute for Health Metrics and Evaluation, 2010) MOI were timely in filling death notification forms but the qualified medical officers showed little concern on timing in filling DNF. The MOI are under supervision and so their work is likely to be timely.

In the study regression analysis results revealed that hospital where the records are reported $(p=0,001)$, qualifications of the certifier of forms $(\mathrm{p}=0.022)$, age of the deceased $(\mathrm{p}=0.014)$, gender of the deceased $(0.015)$, underlying cause of death (0.001), intermediate cause of death (0.001), immediate cause of death $(0.001)$ and source of the information used to fill the death notification forms $(0.001)$ has a statistically significant relationship with completeness of the death notification forms. This study shows the clinician cadre had a statistical significance on completeness of the DNF a fact alluded to by studies by Institute of health metrics (WHO, 2000). However in this study a majority of the health workers were MOI explaining the high incidence rate. Department of paediatrics had the least errors indicating that clinicians in this department were more focused on their work. This can be attributed to the Millennium development goals of reduction of child mortality by a third and emphasis put on any child mortality. Most of the DNF never had the source of filling it in that health workers never reported whether they attended to the patient before death, examined the body or performed post mortem. This raises questions on the authenticity and validly of information therein. With missing source of information the completeness of DNF can't be achieved as demonstrated in a study (Jamal et al, 2017) whose findings were at 99\% incorrect clinical sequence of death. Results indicated that out of the 51 clinicians studied, $78 \%$ had attended pre-training on filling a death notification forms. A higher proportion of the clinicians who reported to have formal pre-training (75\%) reported good knowledge on filling the forms compared to the $64 \%$ of the clinicians who said they had no pretraining on the filling of forms. It's therefore evidenced that pre training had direct impact on knowledge of filling the DNF. However, higher proportion of clinicians who had not attended a pre-training (27\%) had excellent knowledge compared to the $13 \%$ of the pre-trained clinicians who had excellent knowledge. This illustrates that on job and mentorship on filling the DNF improves efficiency on filling this vital document in the event of death

\section{Conclusion}

This study concluded that DNF is not completed comprehensively and it is not done at the threshold of 48 hours required by law. This conclusion necessities a review of policy to increase the professionals permitted to certify death, training of hospital staff certifying death and regularity of sensitization.

\section{Acknowledgements}

The authors are grateful to the personnel at Thika Level Five and Maragua Level Four Hospitals for providing data and interview time for this study.

Declaration of Conflict. The authors declare no conflict of interest

\section{References}

Institute for Health Metrics and Evaluation, (2010) Analyze updated data about the world's health levels and trends from 1990 to 2017 in this interactive tool using estimates from the Global Burden of Disease (GBD) 
Institute $\frac{\text { study. }}{\text { for }}$ Health Metrics and Evaluation. (2010, May 10). Retrieved from https://en.wikipedia.org/wiki/Institute for Health Metrics and Evaluation

Jamal (2017). Department of paediatrics Quality of death notification forms in North West Bank/Palestine:

Jamal, A. S., Nazzal, Z., Yacoup, A. R., \& Mansour, M. (2017). Quality of death notification forms in North West Bank/Palestine: a descriptive study. BMC Research Notes, 10(1).

Katsakiori, P., Liatsikos, E. N., \&Stolzenburg, J. (2007). Haemostasis in Radical Prostatectomy. Endoscopic Extraperitoneal Radical Prostatectomy, 135-142. doi:10.1007/978-3-540-45536-3_9

MCAllum. (2007, October 19). Disease and the dynamics of extinction Philosophical Transactions of the Royal Society B: Biological Sciences. https://royalsocietypublishing.org/doi/abs/10.1098/rstb.2012.02.

Patel, M., Swadia, V., \& Bansal, G. (2010). Prospective randomized comparative study of use of PLMA and ET tube for airway management in children under general anaesthesia. Indian Journal of Anaesthesia, 54(2), 109. doi:10.4103/0019-5049.63643

United Nations, (2004). Development Programme. Human Development Report, 2004: Cultural Liberty in Today's Diverse World. New York: UNDP.

WHO. (2017). World Health Statistics 2017: Monitoring health for the SDGs. Retrieved from https://www.who.int/gho/publications/world health_statistics/2017/en/

World Health Organization (2000). The world Health Report 2000 Health Report 2000 Health Systems: Improving performance. Geneva: WHO

Yudkin et al, (2009) Reflexive intergroup bias in third-party punishment, Journal of Experimental Psychology: General 145:1448-1459.

Yudkin, P. L., Burger, E. H., Bradshaw, D., Groenewald, P., Ward, A. M., \&Volmink, J. (2009). Deaths caused by HIV disease under-reported in South Africa. AIDS, 23(12), 1600-1602.

ZaherNazzal. (2017). Quality of death notification forms in North West Bank/Palestine: a descriptive study. Retrieved from https://www.ncbi.nlm.nih. gov/pmc/articles/ PMC5387246/ 